EUROPEAN ORGANIZATION FOR NUCLEAR RESEARCH 843408

CERN - AT DIVISION

$C_{i}$

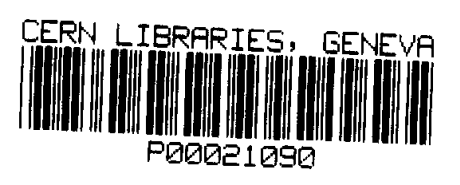

CERN AT/93-57 (MA)

LHC Note 261

Status of the Fabrication and Test of the Prototype LHC Lattice Quadrupole Magnets

N. Siegel, T. Tortschanoff

J.M. Rifflet*, P. Giovannoni*, F. Le Coz*, C. Lyraud*, J. Perot*, P. Védrine*

\begin{abstract}
Within the framework of the Large Hadron Collider (LHC) $\mathrm{R} \& \mathrm{D}$ programme, CERN and CEN/Saclay have established a collaboration to carry out, amongst others, the design, building, and testing of a superconducting LHC prototype quadrupole at the Saclay laboratory. The twin aperture prototype, working in liquid helium at $1.8 \mathrm{~K}$, has a gradient of $250 \mathrm{~T} / \mathrm{m}$, is about $3.2 \mathrm{~m}$ long, with a coil aperture of $56 \mathrm{~mm}$. This paper describes the present state of the $R \& D$ work for the quadrupole magnet, reviewing the fabrication and validation tests of the design.
\end{abstract}

* CEN/Saclay

13th International Conference on Magnet Technology (MT13), Victoria, Canada 20-24 September 1993

Geneva, Switzerland

02/01/94 


\title{
Status of the Fabrication and Test of the Prototype LHC Lattice Quadrupole Magnets
}

\author{
J.M. Rifflet, P. Giovannoni, F. Le Coz, C. Lyraud, J. Perot, P. Védrine \\ CEN/Saclay-DSM/DAPNIA/STCM \\ 91191 Gif-sur-yvette France \\ N. Siegel, T. Tortschanoff \\ CERN/AT/MA \\ 1211 Geneva 23, Switzerland
}

\begin{abstract}
Within the framework of the Large Hadron Collider (LHC) R\&D program, CERN and CEN/Saclay have established a collaboration to carry out, amongst others, the design, building, and testing of a superconducting LHC prototype quadrupole at the Saclay laboratory. The twin aperture prototype, working in liquid helium at $1.8 \mathrm{~K}$, has a gradient of $250 \mathrm{~T} / \mathrm{m}$, is about $3.2 \mathrm{~m}$ long, with a coil aperture of $56 \mathrm{~mm}$. This paper describes the present state of the $R \& D$ work for the quadrupole magnet, reviewing the fabrication and validation tests of the design.
\end{abstract}

\section{INTRODUCTION}

CEN/Saclay has a long standing tradition in collaborating and carrying out experiments and equipments with other laboratories working in the High Energy Physic field, and in particular with CERN. Since 1989, a collaboration has been etablished between CERN and CEN/Saclay in the frame of CERN's R\&D effort to build superconducting magnets for the LHC project [1].

The work at Saclay consists of:

- detailed design of the LHC lattice quadrupoles.

- drawings of the twin aperture cold mass

- design, specification and follow-up of the fabrication in industry of the tooling necessary to assemble the quadrupole cold mass.

- fabrication of one single quadrupole with dummy cable (collared coils).

- fabrication of two superconducting cold masses.

- cryogenic and electric test of these two cold masses.

- writing technical documents (process description and travelers) in view of a later industrial series production of the quadrupoles.

Manuscript recelved September 20,1993

\section{BRIEF DESCRIPTION OF THE MAGNET}

Reference [1] gives a full description of the magnet. We then just list the main parameters of the magnet in table 1 below.

TABLE 1

MAIN PARAMETERS OF THE PROTOTYPE MAGNET

\begin{tabular}{lr} 
Nominal gradient $(\mathrm{T} / \mathrm{m})$ & 252 \\
Inner coil aperture $(\mathrm{mm})$ & 56 \\
Magnetic length $(\mathrm{m})$ & 3.055 \\
Overall current density $\left(\mathrm{A} / \mathrm{mm}^{2}\right)$ & 530 \\
Peak field (T) & 7.76 \\
Nominal current $(\mathrm{A})$ & 15060 \\
Magnetic force Fx per octant $(\mathrm{kN} / \mathrm{m})$ & 625 \\
Magnetic force Fy per octant $(\mathrm{kN} / \mathrm{m})$ & $-\mathbf{8 8 0}$ \\
Stored energy in the cold mass $(\mathrm{kJ})$ & 890 \\
\hline
\end{tabular}

\section{DESCRIPTION OF THE FABRICATION}

A. The coil.

The coil is wound with a keystoned cable made of 24 strands and the bare cable dimensions are (1.70-2.16) $\mathrm{mm} \times 13.05 \mathrm{~mm}$. There are 20 turns (8 in the first layer with a spacer and 12 in the second layer without spacer). The second layer is wound on top of the first one without any splice in order to reduce the heat generated by joule effect. This option implies that the first layer is polymerized twice. Test on samples [2] have shown that a second polymerization cycle does not modify significantly neither the dimensional nor the mechanical properties (Young modulus) of the coil.

It is well known that magnet coils must be under adequate prestress to withstand the magnetic forces without quenching. This is generally obtained by adding shims to the coils during the collaring but has the disadvantage of affecting the magnetic field homogeneity. It was therefore preferred not to shim the coils during assembly but to shim the polymerisation mould depending on the measured mechanical and dimensional behaviours of a polymerised sample of stacked conductors. This procedure is fully described in [3]. This resulted in 
coils which have the required prestress with good azimutal dimensions.

The ramp of the conductor between the first and the second layer, located at the end of the straight part of the coil, has to be as short as possible in order to reduce the perturbation of the magnetic field, however the section of the cable, and consequently its rigidity, is relatively high. The length of this ramp has been fixed to $70 \mathrm{~mm}$. During the winding of 5 dummy coils the way to form the ramp has been improved: The best way is to manually form the insulated conductor and to glue it on the head spacer with epoxy resin.

After delivery of the superconducting cable, it was found out that its mechanical behaviour under compression was very different from that of the dummy one althought the bare dimensions were correct. This is due to the filling factor which is $88,6 \%$ for the dummy and $93 \%$ for the superconducting cable. It resulted that the polymerisation press was not strong enough to bring the coil to its right dimensions, which required to reduce the thickness of the kapton insulation from $75 \mu \mathrm{m}$ ( $25 \mu \mathrm{m}$ with $48 \%$ overlap +12.5 $\mu \mathrm{m}$ with $48 \%$ overlap) to $62.5 \mu \mathrm{m}$ (12.5 $\mu \mathrm{m}$ with $48 \%$ overlap $+12.5 \mu \mathrm{m}$ with $60 \%$ overlap)

Unfortunately, this modification induced a more brittle insulation and the first collaring revealed short-circuits in two coils wich were found to be caused by the breaking of the insulation in the coil head under a local overpressure. The problem was solved by modifying the shape of the head spacers. This also lead to a modification of the control procedure: in addition to the planned electrical tests (i.e. ohmic resistance, self inductance and high voltage test consisting in discharging capacitors charged with 1000 volts) the ohmic resistance and the high voltage test were also performed with the heads of the coil submitted to an azimuthal pressure of $50 \mathrm{MPa}$.

The head spacers have been obtained in the following way:

First, a sheet of metal representing one side of the cable is formed on a tooling whith the following limit conditions: exact angles and locations at the end of the straight part of the coil and in the mid plane of the spacer. Inside this form, a modelpiece is moulded using a suitable moulding resin. Finally, the head spacer is machined out of a tube of glass epoxy composite with a spherical milling head by copying the model.

Because the sheet has a high rigidity in its own plane, it can not react like the cable. Therefore this method is not very accurate and it can explain the overpressures mentioned above.

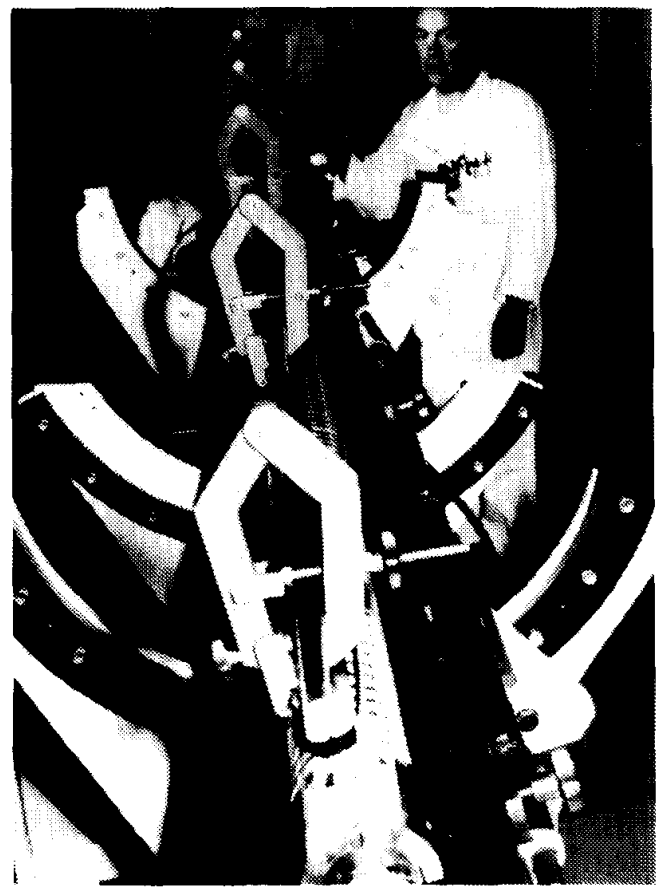

Fig. 1. The Coil Winding

In the future we plan to machine the head spacers directly from geometries coming from magnetical calculations with a computer driven milling machine. This method will be tested before the end of 1993 on the quadrupoles for the high energy booster for SSC [4].

The fabrication of the coils in now finished. In total, 26 coils (5 with dummy cable and 21 with superconductor) have been produced. Fig. 1 shows one of these coils under winding. The winding machine and the polymerisation press are ready for industrial production.

\section{B. The coil collaring.}

The way to assemble the coils with stainless steel collars and tapered keys [1] was a challenge. Tests have been carried out to verify if the method to determine the dimensions of the polymerisation mold, in combination with the collaring process leads to the correct prestress in the coil (50 to $65 \mathrm{MPa}$ measured for a minimum target value of $40 \mathrm{MPa}$ ). These tests are described in detail in [3] and they show that the goal has been achieved.

The collaring procedure works quite well, but some improvements remain to be done before going to industrial production. During the collaring of the quadrupoles of the first cold mass, special collars equiped with strain sensors have been inserted in order to follow the changes in prestress during cool down and 
energization. Five single quadrupoles have been collared ( 1 dummy and 4 superconducting). Fig. 2. shows a picture of one of these single quadrupoles

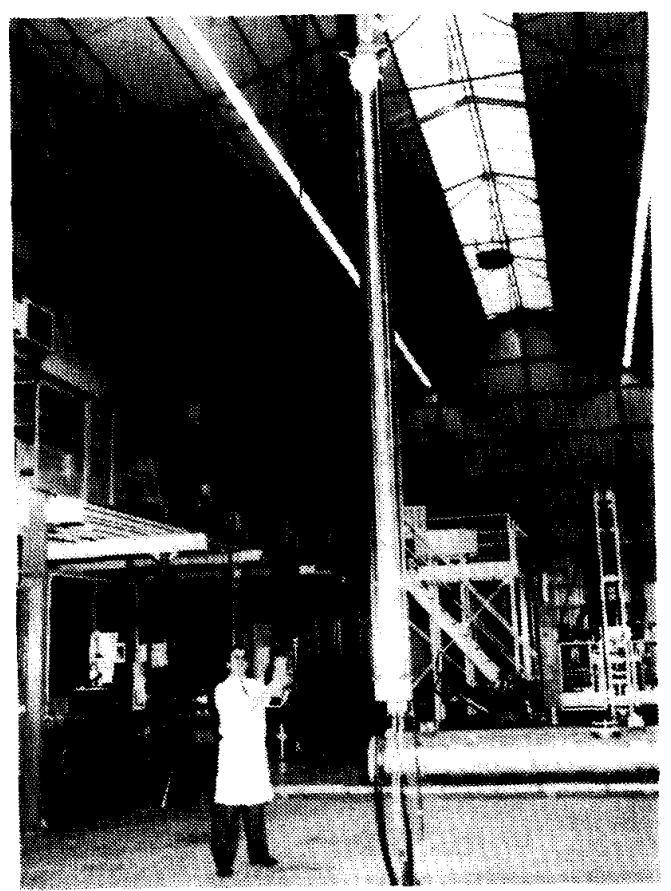

Fig. 2. A single quadrupole after collaring

After collaring the electrical tests (ohmic resistance, self-inductance, verification of insulation between coils $(1000 \mathrm{~V})$ and to ground $(5000 \mathrm{~V}))$, the warm magnetic measurements are performed. Table 2 gives the results of these measurements made on the dummy single quadrupole and on the 2 quadrupoles of the second cold mass. These results are at present under investigation at CERN [5], where inverse calculations should permit to detect some systematic errors in the tooling. The two superconducting magnets of the second cold mass are within the tolerances.

After warm magnetic measurements, the final electrical connections between coils are made and the potential wires are put in place. The keys are welded to the packing strips to avoid them to slip out and thus decrease the prestress. The single quadrupole is ready for the iron stacking operation once the electrical tests (ohmic resitance, self-inductance, verification of ground insulation $(5000 \mathrm{~V})$ ) and the mounting of temperature sensors are finished.

To complete the work, there is still 1 single quadrupole to assemble (a spare one). It will be collared after testing the coils with the Young modulus measurement device which will become available at the end of 1993 .
TABLE 2

Results of magnetic measurements $\triangle B / B$ in $10^{-4}$ at $r=15 \mathrm{~mm}$

$\mathrm{n}=3$ is the sextupole

\begin{tabular}{lrrrr}
\hline n & dummy & Q21 & Q22 & Spec \\
\hline \multicolumn{5}{c}{ normal terms } \\
\hline 3 & 2.52 & -0.25 & -0.90 & $0 \pm 3.29$ \\
4 & 0.13 & 0.16 & 0.33 & $0 \pm 1.48$ \\
5 & -0.45 & 0.33 & 0.10 & $0 \pm 1.39$ \\
6 & 0.04 & 0.35 & 0.03 & $-0.06 \pm 1.45$ \\
7 & -0.04 & -0.09 & 0.00 & $0 . \pm 0.24$ \\
8 & 0.01 & 0.01 & 0.00 & $0 . \pm 0.33$ \\
9 & 0.01 & 0.02 & 0.02 & $0 . \pm 0.51$ \\
10 & -0.23 & -0.29 & -0.27 & $-0.25 \pm 0.25$ \\
\hline \multicolumn{5}{c}{$-5 k e w$ terms } \\
\hline 3 & -2.42 & 2.49 & $0 . \pm 3.69$ \\
4 & -0.74 & 0.14 & 0.42 & $0 . \pm 1.57$ \\
5 & 1.29 & -0.25 & 0.20 & $0 . \pm 0.68$ \\
6 & -0.36 & -0.04 & -0.07 & $0 . \pm 1.10$ \\
7 & -0.03 & -0.03 & 0.13 & $0 . \pm 0.24$ \\
8 & -0.01 & -0.02 & -0.05 & $0 . \pm 0.33$ \\
9 & 0.00 & -0.03 & 0.01 & $0 . \pm 0.10$ \\
10 & 0.01 & 0.00 & 0.02 & $0 . \pm 0.10$ \\
\hline
\end{tabular}

\section{The iron stacking and cold mass assembly}

The iron stacking procedure was another challenge [1]: Batches of non-split iron laminations are brought sliding down along the two single quadrupoles standing up right, then they are pushed laterally to center the two quadrupoles with respect to the laminations and finally they are locked together This operation seemed to be difficult but in fact, it is perhaps the easiest one in all the cold mass assembly. At the design time, it was planned to slide down batches of 2 laminations ( $10 \mathrm{~mm}$ ) at a time, in reality, we used batches of 6 laminations $(30 \mathrm{~mm})$ for the first cold mass and batches of 10 laminations $(50 \mathrm{~mm})$ for the second one.

Somewhat more difficult is the installation of the busbars. They are inserted in grooves made at the outside of the iron core and are relatively heavy. A simple tooling holding the busbars while they are being inserted would facilitate this operation.

Once the busbars are in place, the inertia tube is lowered and the centering keys of the magnetic core are locked. No difficulties were encountered in the locking operation even if, at the beginning, the magnetic core is off centred by a few tens of $\mathrm{mm}$. The cold mass is then turned horizontal after which the electrical connections between coils and busbars are done. Fig. 3. shows the cold mass at this stage. 


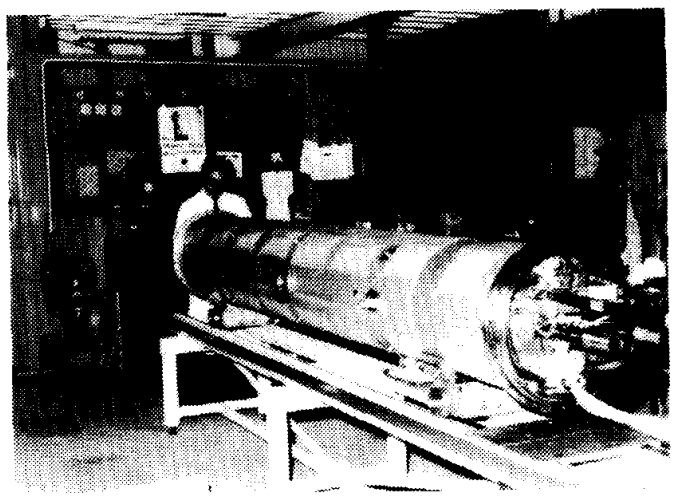

Fig. 3. The cold mass before the helium vessel is closed

After the beam tubes are installed, the tightness side-caps and the end caps are welded. Once the temperature, pressure and displacement sensors (between each single quadrupole and the iron core and between the iron core and the inertia tube) are put in place and electrical tests (ohmic resistance, staging of potential wires, insulation of the magnets with respect to ground $(3000 \mathrm{~V})$, verification of all sensors wires) and tightness check are finished, the cold mass is ready to be inserted in the cryomagnetic test facility [6] shown in Fig 4

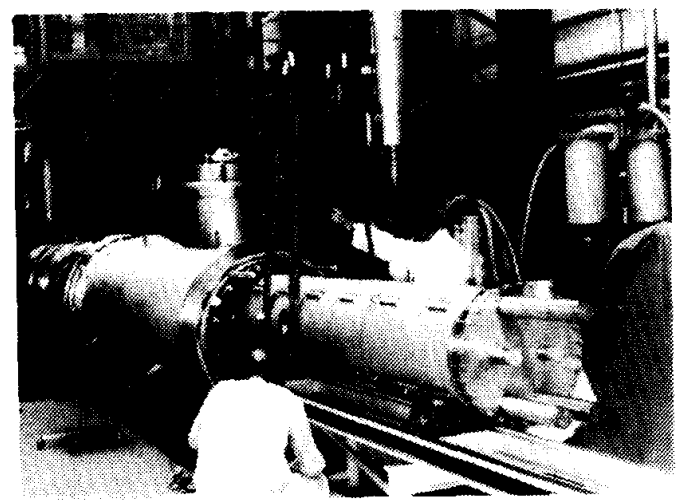

Fig. 4. Insertion of the first cold mass in the Saclay's test facility

\section{CONCLUSION}

Two coldmasses have been assembled and the fabrication process is mastered. For the series fabrication, only a few details remain to be improved.

The first cold mass is installed in the cryogenic test facility at Saclay and will be cold tested in October 1993

The design was performed on parameters which were frozen in July 1990. Since this date, the parameters of the LHC machine have changed and will be fixed at the end of 1993 . The gradient will be lowered (to about $220 \mathrm{~T} / \mathrm{m}$ ), and the current will be reduced. A new design must then be undertaken and the tooling should be adapted to this new parameters (only minor changes are expected). A new cold mass prototype will probably be built (discussions are in course) before finalizing the specifications for industrial series production.

\section{ACKNOWLEDGMENTS}

The authors would like to thank all their colleagues from CERN and CEA/Saclay who have participated to the design, fabrication and tests of the LHC lattice quadrupole prototype.

\section{REFERENCES}

[1] J.M. Baze et al.,"Design and fabrication of the Protoype Superconducting quadrupole for the CERN LHC Project", Proceedings of MT-12 conference, IEEE Trans. Magn., vol. 28; p. 335, June, 1991.

[2] R. Gallet, G. Lemiere, P. Vedrine, "Détermination des dimensions du moule de polymérisation", Internal report CEA/DSM/DAPNIA/STCM 102 02R 235 TC 51.

B] P. Vedrine et al., "Mechanical tests on the Prototype LHC Lattice Quadrupole", submited to this conference, paper $\mathrm{N}^{\circ}$ 108.

44] G. Ducos et al., "Design and main Parameters of the High Energy Booster Quadrupole cold Mass for the SSC", submited to Fifth Annual International Industrial Symposium on the Super Collider (IISSC) - San Francisco, USA - may 1993

[5] S. Russenschuck at al., "Tracing Back Measured Field Imperfections in LHC Magnets by Mean of the Inverse Problem Approach", submited to this conference, paper $N^{\circ} 124$.

[6] J. Deregel et al., "Station d'essais a $1.8 \mathrm{~K}$ pour aimants d'accelerateurs", Internal report DAPNIA/STCM CRYOMAG/93-05, may 1993 